\title{
Estrategias de la industria tabacalera en México para interferir en las políticas de control del tabaco
}

\author{
Alejandro Madrazo-Lajous, D en Der, (1) Ángela Guerrero-Alcántara. ${ }^{(2)}$
}

\author{
Madrazo-Lajous A, Guerrero-Alcántara A. \\ Estrategias de la industria tabacalera \\ en México para interferir en las políticas \\ de control del tabaco. \\ Salud Publica Mex 20I2;54:3I5-322.
}

\begin{abstract}
Resumen
Objetivo. Identificar las estrategias empleadas por la industria tabacalera en México para contener tanto la adopción del Convenio Marco para el Control de Tabaco como la implementación adecuada de las políticas que contempla. Discusión. La interferencia de la IT en el diseño, adopción e implementación de las políticas de control de tabaco se ha intensificado desde la firma del CMCT. A partir de 1997, las estrategias se adaptaron al cambio político en México. Esta adaptación consistió en identificar los puntos de veto en el desarrollo de las políticas de control de tabaco. Conclusión. Las estrategias de interferencia de la industria tabacalera son eficaces en la afectación de las decisiones públicas.
\end{abstract}

Palabras clave: tabaco; legislación; políticas; México
Madrazo-Lajous A, Guerrero-Alcántara A.

Undue tobacco industry interference

in tobacco control policies

in Mexico.

Salud Publica Mex 20I2;54:3 I5-322.

\begin{abstract}
Objective. To identify tobacco industry's strategies aimed at containing the full adoption of public health policies established by the Framework Convention on Tobacco Control. Discussion. Tobacco industry interference in the design, adoption and implementation of tobacco control policies has intensified since the signing of the FCTC. However, it is back in 1997 when one can trace a shift in tobacco industry strategies, adapting to political change in Mexico. This adaptation has consisted mostly in identifying emerging veto points in the chain of public policy development. Conclusion. Tobacco industry's interfering strategies have success y fully affected Mexican policies.
\end{abstract}

Keywords: tobacco industry; drug control; health policy; lobbying

Este documento presenta una síntesis de la investigación realizada en el Programa de Derecho a la Salud del Centro de Investigación y Docencia Económica (CIDE) sobre las estrategias de interferencia de la IT en México en la aplicación de políticas de control de tabaco y cierra con algunas recomendaciones para hacer frente a las acciones de la IT.

(I) Programa de Derecho a la Salud, División de Estudios Jurídicos del Centro de Investigación y Docencia Económica. México.

(2) Área de Control de Substancias del Programa de Derecho a la Salud del Centro de Investigación y Docencia Económica. México.

Fecha de recibido: 6 de marzo de 2012 Fecha de aceptado: 10 de abril de 2012

Autor de correspondencia: Dr. Alejandro Madrazo Lajous. Centro de Investigación y Docencia Económicas. Circuito Tecnopolo Norte esq. Circuito Tecnopolo Oriente, col. Hacienda Nueva. 20313 Aguascalientes, Aguascalientes, México.

Correo electrónico: Alejandro@madrazolajous.com 
$E^{1}$ 12 de mayo de 2004 el presidente Vicente Fox publicó en el Diario Oficial de la Federación (DOF) el decreto del Senado con el que México ratificó el Convenio Marco para el Control del Tabaco (CMCT). El objetivo del convenio, vigente desde entonces, consistió en comprometer a los países signatarios a instrumentar políticas públicas encaminadas a controlar la epidemia mundial de tabaquismo. ${ }^{1}$ Por su parte, la industria tabacalera (IT) ha desplegado diversas estrategias para entorpecer la aplicación del CMCT en distintos países. ${ }^{2}$ América Latina ha sido una región relevante en este proceso, pues el tamaño actual del mercado, su potencial de crecimiento y la debilidad estructural de las instituciones estatales de la región permiten que las estrategias de la IT sean más efectivas y redituables que las desplegadas en las economías desarrolladas. ${ }^{3}$

Dada la importancia del mercado nacional del tabaco, México ha sido uno de los países prioritarios para la IT internacional. ${ }^{4}$ La implementación cabal del CMCT en nuestro país resulta estratégica tanto para la región como para el esfuerzo mundial por consolidar políticas de control del tabaco. ${ }^{5}$

Las políticas previstas por el CMCT no han sido cabalmente implementadas, inclusive en su versión mínima, a pesar de haber transcurrido más de siete años desde que México ratificó el $\mathrm{CMCT}^{*}$ a la fecha. ${ }^{6}$ La interferencia de la IT se ha intensificado a partir de la firma del CMCT en 2003. Ya desde 1997, cuando por primera ocasión el partido en el gobierno perdió la mayoría legislativa, la IT se había adaptado al cambio político en México al identificar los nuevos puntos de veto en la cadena de elaboración de las políticas públicas. ${ }^{7}$ A partir de entonces, la IT ha desplegado múltiples estrategias para influir en el diseño de la política de control de tabaco. ${ }^{7}$ De este modo, ha conseguido alterar y retrasar la promulgación de un marco regulatorio que adopte plenamente las políticas del CMCT y sirva como fundamento para una política pública eficaz en esta materia.

Los resultados de la investigación se lograron a partir de un estudio cualitativo donde se detalla la identificación de actores, conceptos y variables clave necesarios para comprender en qué consisten los instrumentos de control de tabaco. Se identificaron cinco grupos clave en materia de control de tabaco: 1) cabilderos, 2) representantes de organizaciones civiles, 3) funcionarios públicos a favor del control de tabaco, 4) funcionarios públicos en contra del control de tabaco y 5) asesores jurídicos. Una

*México firmó el tratado en 2003 y lo ratificó en 2004. vez ubicados, se construyeron cuestionarios semiestructurados para cada una de las categorías encontradas con tres grupos de información: 1) actividad que realiza, 2) relación con la IT o el gobierno, 3) impacto de las estrategias de interferencia y recomendaciones.

Posteriormente, se recabó información y se realizó una revisión bibliográfica sobre las estrategias de interferencia de la IT en otros países. Se revisaron convenios internacionales firmados y ratificados por México; se rastreó la modificación del marco regulatorio mexicano en materia de impuestos al tabaco y de legislación de salud y se consultaron los archivos de las gacetas parlamentarias de las dos cámaras legislativas a nivel federal. Terminado este proceso, se realizaron diez entrevistas confidenciales a legisladores, integrantes de la sociedad civil y cabilderos de la IT. Se identificaron los tipos, las esferas de incidencia y el número de estrategias que la IT ha desplegado durante la última década. De esta forma, se definieron las estrategias de la IT sobre las instituciones del Estado mexicano y cómo respondió éste a los objetivos de la industria. Finalmente se analizaron los resultados obtenidos y se exploraron las implicaciones políticas y sociales derivadas de la investigación en este último sexenio.

Dada la naturaleza no pública de muchas de las actividades relacionadas con el cabildeo y las estrategias de la IT, es difícil generar datos que permitan conocer con amplitud los alcances y las características de dichas actividades, por lo que en parte éstas se han inferido. El lector debe tomar en consideración este aspecto.

\section{Discusión}

Se identificaron cinco momentos clave para el control de tabaco en México entre los años 2004 y 2010. Cada uno constituye un proceso en el cual han intervenido diversos actores formales (institucionales) e informales (actores independientes o individuales) en un periodo determinado de tiempo. Los momentos son: a) La firma del Convenio Marco de la Organización Mundial de la Salud para el Control del Tabaco en 2003, y la ratificación del mismo en 2004; b) El acuerdo entre el poder Ejecutivo federal y la IT comúnmente conocido como "un peso por cajetilla", firmado en 2004, el cual fijaba por mutuo acuerdo la regulación de los productos derivados del tabaco y establecía el acuerdo común de evitar la imposición de nuevos o mayores impuestos al tabaco. ${ }^{8}$ c) La aprobación y promulgación de la Ley General para el Control del Tabaco (en adelante, LGCT) en 2008 que contenía, entre otras, las siguientes medidas: la prohibición para la industria de regalar y vender cigarros en unidades sueltas; la separación 
física -aislamiento mediante barreras físicas y sistemas de ventilación- entre espacios de fumadores y no fumadores en espacios públicos y la prohibición de fumar en áreas de trabajo; la inclusión de pictogramas en las cajetillas de cigarros en $30 \%$ de la cara anterior; la restricción -pero no la eliminación- de la publicidad del tabaco entre otras; d) La aprobación de un esquema de alza gradual de impuestos establecido en la Ley del Impuesto Especial sobre Producción y Servicios (en adelante, IEPS), en 2009, y e) el aumento adicional en el precio de las cajetillas para 2011 el cual correspondió a un incremento del Impuesto Especial sobre Producción y Servicios (el aumento fue de cinco pesos, que aunados a los dos pesos graduales suman siete pesos para las cajetillas de 20 cigarros).

\section{Áreas prioritarias para la IT en México}

Los documentos internos de la IT permiten identificar que las compañías tabacaleras se han preocupado por los mismos temas en todo el mundo. Consistentemente, la IT busca evitar la implementación de políticas públicas orientadas a disminuir o desalentar el consumo de tabaco. En su defecto, procura la adopción de las políticas de control de tabaco menos eficaces en la disminución del consumo.

A partir de los planes e informes de las empresas más grandes que operan en Latinoamérica -British American Tobacco (BAT) y Philip Morris México (PMM)- se pueden inferir los rubros prioritarios para la IT de política de control de tabaco. Éstos se agrupan en los siguientes rubros: 1) tributación, 2) libertad de comercio, 3) espacios 100\% libres de humo, consumo de tabaco y salud y 4) publicidad y relaciones públicas. En el cuadro I se observa la importancia de cada rubro y los objetivos de la IT:

Las dos áreas más importantes son tributación y libertad de comercio (publicidad). En la primera se busca que los impuestos se mantengan en el nivel más bajo posible como porcentaje del precio final al consumidor $\mathrm{o}$, en su defecto, que el aumento en impuestos sea gradual y no sea ad valorem. ${ }^{5}$ Para la industria los efectos en el alza de impuestos son equiparables a la aplicación del conjunto del resto de las políticas de regulación. *

En el caso de la libertad de comercio, busca aprovechar al máximo todas las herramientas que, directa o indirectamente, le permitan llegar más fácilmente a todos los segmentos de mercado: adolescentes, jóvenes,

\footnotetext{
* Ángela Guerrero, Entrevistado 6. México DF, mayo 2010.
}

mujeres y adultos. En las otras áreas, el impacto de las políticas en el mercado de la IT son indirectas por lo que no son consideradas tan importantes y, en consecuencia, son utilizadas como "fichas de cambio" para lograr una negociación con los tomadores de decisión. En palabras de un legislador entrevistado confidencialmente: "las tabacaleras están dispuestas a hablar de algunos temas que impliquen su regularización, mientras no involucre un tema tributario". ${ }^{*}$ Es fundamental tener en mente este punto pues de él se desprende una de las recomendaciones que resulten quizá más relevantes para impulsar exitosamente políticas de control de tabaco, como se verá en las conclusiones.

\section{Identificación de las estrategias de la IT}

El objetivo general de la IT consiste en evitar o retrasar la entrada en vigor de cualquier política pública de control de tabaco por medio de estrategias que construyan y fortalezcan relaciones políticas con actores claves. Aunque en México se conoce el intenso trabajo que realizan despachos de cabildeo, su actividad ha sido regulada mínimamente. Hasta septiembre de 2010 entró en vigor el reglamento de la Cámara de Senadores y en enero de 2011 el de la Cámara de Diputados. ${ }^{9}$ En ambos casos se establece qué es el cabildeo y se obliga a los legisladores a publicar las actividades que realizan con los cabilderos. Resulta ilustrativo de las estrategias de la IT que al registrar a los cabilderos en la Cámara de Diputados, la Mesa Directiva informó que PMM registró seis de ellos. ${ }^{10}$

El cabildeo se puede clasificar en dos tipos: cotidiano y estratégico. El cabildeo cotidiano se realiza principalmente en el Poder Legislativo por cabilderos oficiales que están diariamente en las Cámaras. Sus principales funciones son: 1) servir de interlocutores entre la empresa y diputados y senadores; 2) hacer llegar a los legisladores información relevante para la industria, 3) iniciar y estrechar lazos con los principales dirigentes. Un ex ejecutivo de la IT en entrevista comentó: "un cabildero todo el tiempo construye relaciones porque no sabes en qué momento vas a requerir tener acceso a cierto actor de política pública, siempre necesitas estar abriendo canales de comunicación que eventualmente se necesitarán".:

El cabildeo estratégico consiste en buscar negociaciones coyunturales entre la IT y actores políticos claves

* Ángela Guerrero, Entrevistado 1. México DF, mayo 2010.

‡ Ángela Guerrero, Entrevistado 6. México DF, junio 2010. 


\begin{tabular}{|c|c|c|c|}
\hline \multicolumn{4}{|c|}{ ITARIAS PARA LA INDUSTRIA TABAC } \\
\hline $\begin{array}{l}\text { Área de control } \\
\text { de tabaco }\end{array}$ & $\begin{array}{l}\text { Efectividad de la intervención } \\
\text { para combatir el tabaquismo }\end{array}$ & $\begin{array}{l}\text { Objetivos generales } \\
\text { de interferencia de la IT. }\end{array}$ & $\begin{array}{l}\text { Objetivos específicos de } \\
\text { interferencia de la IT. }\end{array}$ \\
\hline Tributación & Muy alta & $\begin{array}{l}\text { Mantener la tasa del impuesto al tabaco, en } \\
\text { términos del precio final al consumidor, tan } \\
\text { baja como sea posible, idealmente, menor } \\
\text { al } 75 \% \text {. }\end{array}$ & $\begin{array}{l}\text { Gradualidad, sobre alzas abruptas de impuestos. } \\
\text { Impuestos nominales sobre los ad valorem. } \\
\text { Diferenciación de tasas impositivas para cigarros } \\
\text { con filtro y sin filtro. }\end{array}$ \\
\hline $\begin{array}{l}\text { Libertad de } \\
\text { comercialización }\end{array}$ & Alta & $\begin{array}{l}\text { Mínimas restricciones a la comercialización } \\
\text { de cigarros }\end{array}$ & $\begin{array}{l}\text { Inexistencia de requerimientos legales para contar } \\
\text { con licencias sanitarias para vender cigarros. } \\
\text { Falta de un registro de puntos de venta. } \\
\text { Posibilidad de vender cigarros sueltos. }\end{array}$ \\
\hline $\begin{array}{l}\text { Espacios } 100 \% \\
\text { libres de humo de } \\
\text { tabaco (Humo de } \\
\text { segunda mano) }\end{array}$ & Alta & $\begin{array}{l}\text { Evitar que existan áreas públicas } 100 \% \text { libres } \\
\text { de humo de tabaco }\end{array}$ & $\begin{array}{l}\text { Que los negocios cuenten con áreas exclusivas } \\
\text { para fumar. } \\
\text { Que en las áreas exclusivas para fumar pueda } \\
\text { acceder personal de servicio. } \\
\text { Equiparar los derechos de los fumadores a los } \\
\text { no fumadores. }\end{array}$ \\
\hline $\begin{array}{l}\text { Advertencias } \\
\text { sanitarias }\end{array}$ & Alta & $\begin{array}{l}\text { Retrasar la inclusión de advertencias de } \\
\text { riesgos a la salud en las cajetillas }\end{array}$ & $\begin{array}{l}\text { Gradualidad en la adopción de las leyendas de } \\
\text { advertencia. } \\
\text { Utilizar mensajes de texto poco claros en lugar } \\
\text { de imágenes de advertencias. } \\
\text { Otorgar el mínimo espacio en la cajetilla a la } \\
\text { impresión de tales leyendas. }\end{array}$ \\
\hline Publicidad & Media & $\begin{array}{l}\text { Poder utilizar cualquier medio de comunica- } \\
\text { ción para promover el consumo de tabaco. }\end{array}$ & $\begin{array}{l}\text { Restricciones a la publicidad no totales: en par- } \\
\text { ticular poder utilizar revistas "para adultos" y el } \\
\text { servicio postal. } \\
\text { Restricción gradual y autorregulada en medios } \\
\text { de comunicación. } \\
\text { Poder organizar eventos culturales }\end{array}$ \\
\hline
\end{tabular}

A pesar de que esta categoría fue ordenada como "alta" dentro de las entrevistas realizadas a cabilderos de la industria, el tabaco y la salud no fue mencionado como un tema prioritario

tanto del poder ejecutivo como del legislativo. Éste se despliega a partir del trabajo realizado en el cabildeo cotidiano.

En la investigación se identificaron once estrategias de la IT desplegadas con este fin, las cuales se agrupan en dos tipos: las de carácter formal, que consisten en la utilización de las herramientas legales que le permitan a la IT negociar con el Estado sin recurrir abiertamente a la corrupción de los actores, y las de carácter informal, que consisten en la utilización del poder económico de la industria para intimidar y capturar a los actores clave del diseño de la política. El corazón de la estrategia en el segundo grupo radica en acciones que no podrían ser rastreadas ni verificadas en medios de comunicación ni en documentos oficiales del Estado, pero que explican sustantivamente el resultado visible al público.

Las estrategias formales son: 
1. Promover programas que permitan posicionar a las empresas de la IT como empresas socialmente responsables. Entre ellas destaca el pago temprano de impuestos. El impuesto especial sobre producción y servicios recaudado en 2008 superó los 250000 millones de pesos, de los cuales 25500 millones de pesos correspondieron al rubro de tabaco. Así, el ingreso fiscal por venta de tabaco representa $10 \%$ de la recaudación total del IEPS. ${ }^{11}$

2. Regionalizar la problemática de la regulación al tabaco: las empresas tabacaleras pretenden hacer ver que la problemática de la industria es también de los estados donde se realizan actividades económicas vinculadas con la IT, como el cultivo y la fabricación. Aquí, la IT difunde la idea de que en la medida en que se aumenten impuestos en comercio, venta y producción, ellos tendrán que dejar de realizar actividades económicas en los estados donde actualmente residen, lo que "hará perder muchos empleos a nivel estatal". Ejemplo de este discurso es la declaración emitida por un legislador en 2009 durante la discusión del Artículo 4 transitorio de la Ley del Impuesto Especial sobre Producción y Servicios para el año 2010, relativa a tabacos labrados enajenados donde advirtió que "un aumento inmediato al precio por cajetilla hará que se pierdan por lo menos 30000 empleos a lo largo de toda la cadena productiva". ${ }^{12}$

3. Maximizar los costos económicos y políticos de la regulación al tabaco a través de amenazas a la estabilidad económica del país. Éstas se presentan usualmente en dos formas: a) el impacto en la "aportación" tributaria del tabaco para el ingreso fiscal federal y b) el número de empleos que generan en una determinada región del país. La forma en que se despliega esta estrategia es mediante presión en los medios de comunicación, o bien, directamente con los poderes del Estado. Así, por ejemplo, Andrea Martini, director general de ATM, declaró en 2008 en relación con la aprobación de la LGCT: "entre los efectos económicos de la ley, contrasta el pago de 11000 pesos por hectárea cosechada a sus proveedores de tabaco ubicados en Nayarit, contra los 2400 pesos que recibirían al cambiar a la siembra de frijol, por ejemplo. Los trabajadores en el campo que atienden directa o indirectamente las necesidades de BAT suman 6 $568,38 \%$ menos que hace dos años, según cifras de la compañía". ${ }^{13}$

4. Maximización de riesgos ante el aumento de impuestos al amenazar con la posibilidad del contrabando. ${ }^{*}$ La IT sostiene que el aumento en los impuestos trae como consecuencia inmediata un crecimiento del mercado negro y alerta que dicho crecimiento traerá muchos mas problemas de los que el consumo de tabaco a través del mercado formal en México conlleva. ${ }^{14}$ En el marco de esta intervención uno de los entrevistados comentó: "argumentan que va a haber afectación en los estados donde se produce el tabaco, que si se aumenta el impuesto los plantíos de tabaco se convertirán en plantíos de marihuana y que disminuirá el número de empleos". ${ }^{\ddagger}$

5. Difundir estudios "científicos" patrocinados por la industria. Esta estrategia consiste en impulsar o financiar estudios que pretenden demostrar científicamente que el consumo del tabaco no es tan dañino como doctores o asociaciones médicas reconocidas han sostenido. Presentan también investigaciones de corte económico que muestran costos financieros que se generarán a partir de una regulación mayor para la IT. Esto les es muy útil para cualquier mesa de diálogo o negociación. ${ }^{5}$

6. La construcción de acuerdos mutuos o legislación conjunta para intercambiar la aplicación de unos instrumentos de control por otros. Para lograrlo, la IT busca enaltecer la importancia de generar acuerdos integrales en los que sean tomados en cuenta todas las partes interesadas. Ejemplo de ello es la declaración que hizo un legislador al respecto: "en la construcción de la Ley General del Control del Tabaco sostuvimos reuniones con representantes de la industria tabacalera para negociar con ellos todos los temas que queríamos tratar en la Ley para que se pudiera aprobar". Esto a pesar de que el CMCT expresamente establece entre las obligaciones de los Estados parte el evitar que la IT participe en la construcción de políticas de control del tabaco. ${ }^{15}$

Las estrategias informales son:

a) Bloquear o retardar el proceso legislativo para que las iniciativas relevantes no sean dictaminadas o

\footnotetext{
* Después del cierre de la investigación, se presentaron ejemplos de estas estrategias con la aprobación de un aumento de siete pesos a los cigarros por parte de la Cámara de Diputados.

₹ Entrevistado 2. México DF, diciembre 2009.

$\S$ Ángela Guerrero Alcántara y José Sicabí Cruz Salinas, entrevistado 1. México, DF, mayo 2010.
} 
sean rechazadas. * Al respecto un ex ejecutivo de la industria comentó: “...durante las reuniones para la Ley General de Control del Tabaco, hubo un momento donde no nos convenía un texto de la regulación; yo busqué ganar tiempo incluyendo muchas más comisiones de las que la iniciativa debería tener. Lo logré, pero al momento de la votación perdimos ese punto". ${ }^{\ddagger}$

b) Aportar beneficios económicos personales como los regalos y el pago de viajes al extranjero y para el financiamiento de campañas políticas. En ese orden de ideas un legislador comentó: "una tabacalera me envió a mi casa en el mes de diciembre como regalo una caja de puros, la cual no acepté porque excedía el monto permitido, así que a las dos semanas me enviaron sólo seis puros con una constancia notarial de que no excedían el monto permitido para un regalo, y los acepté, pero la caja no". Más $^{\prime}$ adelante puntualizó: "además de los ofrecimientos de dinero que les hacen a los funcionarios, tienen gente permanentemente en las cámaras para evitar cualquier reforma que los afecte".

c) Donar recursos a instituciones de caridad que se estima beneficiarían a actores clave. Otra de las estrategias importantes dentro de este marco es la donación o las ofertas al Estado, situación que se ha dado en distintos momentos. Para ilustrar este punto retomamos lo que un legislador nos comentó: "una vez hubo una tromba muy fuerte en $\mathrm{mi}$ estado y una tabacalera me habló por teléfono para decirme que iba a donar algunas cosas, que ellos

\footnotetext{
* El Poder Legislativo de los Estados Unidos Mexicanos se deposita en un Congreso General, que se divide en dos Cámaras, una de Diputados y otra de Senadores. La formación de leyes y decretos puede iniciarse indistintamente en cualquiera de las dos Cámaras. Toda iniciativa presentada se turna a Comisiones por conducto de la Mesa Directiva para su análisis y posterior dictaminación. Una vez que la comisión elabora el anteproyecto de dictamen, el cual debe ser firmado por la mayoría de los individuos que la compone, se discute en el Pleno de la Cámara y, en su caso, se aprueba. En caso de no ser aprobado, se preguntará en votación económica, si vuelve o no todo el proyecto a la Comisión. Si la resolución fuere afirmativa, volverá para que lo reforme; mas si fuere negativa, se tendrá por desechado. Aprobado un proyecto en la Cámara de origen, pasará para su discusión a la Cámara revisora que recibe la minuta del dictamen con Proyecto de Decreto y lleva a cabo el mismo procedimiento de estudio, dictamen, discusión y aprobación, seguido por la Cámara de origen. Si la Cámara revisora lo aprueba, la ley pasará al Ejecutivo para su publicación.

El presidente de la República, al recibir el decreto aprobado por el Congreso, cuenta con dos opciones: a) realizar observaciones al decreto aprobado, en cuyo caso lo remitirá a la Cámara de origen para su estudio o b) promulgarlo y entonces mandarlo publicar para que se observen y cumplan las disposiciones que contenga la ley.

‡ Entrevistado 8, México DF, junio 2010.

$\S$ Entrevistado 2, México DF, diciembre 2009.
}

depositaban cierta cantidad para que yo pudiera comprarlas. Yo les dije que no podía hacerlo y les recomendé que la donación la hicieran a través de alguna instancia".*

d) Contratar funcionarios de alto perfil en centros de investigación para colocarlos en un conflicto de intereses que les impida promover adecuadamente el control de tabaco. De las entrevistas realizadas se desprende que la IT sostiene, en ocasiones, lo que puede calificarse como contubernio con diferentes actores al interior de los procesos de toma de decisión, en los que se genera un mecanismo de soborno con el cual logran poner en la agenda política la defensa del sector y los temas de su interés. Se tiene registro de diputados que responden directa o indirectamente a los intereses de la IT. Ejemplo de ello es un diputado del cual nos comenta un ex legislador lo siguiente: "quien está asociado con un despacho LTG Lobbying México, que tiene como principal cliente a British American Tobacco, es un legislador muy cercano a un dirigente de un partido político importante del país, es con el cabildeo de ese partido en las dos Cámaras que logran inhibir el impuesto de tajo". . Este testimonio coincide con otros informes sobre el alcance que ha tenido la filtración de las grandes corporaciones en la toma de decisiones públicas, como es el caso del cabildero oficial de FEMSA de 1997 a 2009, tiempo en el que fue legislador suplente en dos ocasiones. ${ }^{16}$

Los esfuerzos de interferencia de la IT se pueden clasificar en función del espacio institucional en el que se despliegan, siendo dos tipos de instancias del Estado mexicano las principales: a) las encargadas de las políticas fiscales -la Secretaría de Hacienda y las comisiones de Hacienda del poder Legislativo federal-y b) las relacionadas con la salud pública -la Secretaría de Salud, las comisiones de Salud del poder Legislativo y la Comisión Federal de Mejora Regulatoria-.

En el primer grupo (instancias fiscales), la IT ha impedido, por largos periodos de tiempo, que el precio de los cigarros aumente lo suficientemente rápido como para reducir la demanda de tabaco. De 2000 a 2009 el aumento en el precio a los consumidores en promedio es de menos de $1 \%$. En las instancias de salud, la industria ha sido capaz de matizar sustancialmente y retrasar la aprobación de leyes y regulaciones sanitarias que protegen la salud de los no fumadores y buscan disminuir la demanda de cigarros a través de intervenciones no relacionadas con los

* Entrevistado 2, México DF, diciembre 2009. 
precios. ${ }^{*}$ Su actuación en ambas instancias le ha permitido disminuir la eficacia del diseño e implementación de la política de control de tabaco en México.

En lo que se refiere a LGCT, la IT logró que se modificara sensiblemente la iniciativa original, lo que permite, entre otras cosas, la creación de áreas exclusivas para fumar en espacios interiores, derrotando así la política recomendada por cuerpos internacionales consistente en establecer espacios totalmente libres de humo de tabaco. Asimismo, en un giro difícil de explicar bajo la normatividad correspondiente al proceso legislativo, el documento aprobado en comisiones fue alterado al someterse al pleno, reduciendo de 100 a 30\% del costado de la cajetilla el espacio asignado a pictogramas para la advertencia sanitaria.

\section{Conclusiones}

Así, las estrategias de interferencia de la IT son, en gran medida, eficaces en su afectación de las decisiones públicas sobre control de tabaco. La investigación emprendida confirma la jerarquía de prioridades de la IT para moldear o frenar la regulación del tabaco y el éxito que ésta ha tenido para influir en nuestra regulación.

La comprensión del modus operandi de la IT ilustra cuán vulnerable es el sistema político mexicano ante industrias tan influyentes como la tabacalera. En la medida en que se identifiquen las estrategias con mayor claridad se logrará reforzar el control de tabaco por dos vías: mediante la obstaculización de las estrategias de la IT y mediante una priorización estratégica de las intervenciones que promueve el movimiento que tome en cuenta la priorización estratégica de la IT.

En este sentido, resultará determinante que quienes impulsan medidas para controlar la epidemia del tabaquismo tengan claras no sólo las prioridades de la IT, sino también las prioridades del movimiento por el control del tabaco y, específicamente, las oportunidades

\footnotetext{
* El proceso de reglamentación de la LGCT estuvo marcado por retrasos en la elaboración de los documentos, modificaciones sustanciales a las disposiciones de la ley, declaraciones de COFEPRIS Y COFEMER, y la intervención de la IT en todo momento. La publicación de la Ley General para el Control del Tabaco por parte del Ejecutivo Federal tomó tres meses después de que había vencido el plazo para ejercer el veto. El reglamento, a su vez, se publicó hasta junio de 2009, más de un año después de la aprobación de la Ley y con más de seis meses de retraso del plazo previsto en ella, y medidas mucho más permisivas y flexibles respecto a los lugares libres de humo, incertidumbre respecto a la publicación de los pictogramas y la vigilancia al cumplimiento de las obligaciones fijadas por la Ley. Para mayor información consultar: http:/ / www.cide.edu/ publicaciones/status/dts/DTEJ\%2051.pdf
}

que ofrece el conocer mejor las prioridades de la IT y utilizar esa jerarquización estratégicamente.

Con los hallazgos presentados, es posible articular, tentativamente, algunas sugerencias a tener en cuenta a la hora de impulsar políticas públicas en esta materia:

1. Conocer las estrategias de "ficha de cambio" que utilizan las tabacaleras, preverlas y contrarrestarlas. En este sentido, se sugiere impulsar distintas medidas de control de tabaco simultáneamente a fin de contar con más "fichas de cambio" al momento de negociar con las tabacaleras, tomando en cuenta la jerarquía de prioridades de la industria tabacalera -referidas en este texto- a la hora del "canje".

2. Difundir información precisa sobre la actividad económica y el impacto del alza de impuestos de la IT a legisladores y funcionarios públicos del poder Ejecutivo así como a la ciudadanía.

3. Realizar periódicamente estudios sobre la identificación de los temas prioritarios para la IT en las políticas de control de tabaco. Identificar las estrategias de interferencia de la IT en el ámbito federal y estatal del poder judicial para conocer las posibles acciones que afecten la eficacia de las políticas públicas adoptadas por las autoridades.

\section{Agradecimientos}

La preparación de este documento fue posible gracias al financiamiento de Tobacco Free Kids para el proyecto "Understanding Undue Tobacco Interference in Tobacco Control Policies in Mexico". En la creación del documento participaron Tania T. Ramírez y José Sicabí Cruz como asistentes de investigación.

Declaración de conflicto de intereses: Los autores declararon no tener conflicto de intereses.

\section{Referencias}

I. World Health Organization, report on the global tobacco epidemic. Ginebra: WHO, 2009.

2. World Health Organization, report on the global tobacco epidemic. Ginebra: WHO, 2008.

3. World Health Organization, report on the global tobacco epidemic. Ginebra: WHO, 2009:5

4. Shafey O, Eriksses M, Ross H, Mackay J. El Atlas del Tabaco. 3ra edición. Atlanta, Georgia: Sociedad Americana del Cáncer, 2009. 5. Aguinaga-Bialous S, Shatensein S. La rentabilidad a costa de la gente. Actividades de la industria tabacalera para comercializar en América Latina y el Caribe y minar la salud pública. Organización Panamericana de la Salud, 2002.

6. López-Antuñano F. Firma y ratificación del Convenio. Salud Publica Mex 2003; 46:155-156. 
7. Immergut $E$. The rules of game: The logic of health policy. En: Steinmo S, Thelen K, Longstreh F, eds. Cambridge: Structuring Politics, 1992:60. 8. Secretaría de Salud. Convenio para ampliar la regulación y normatividad relativas al taba, y el convenio para establecer restricciones adicionales a la regulación y legislación vigente para la publicidad, comercialización y leyendas de advertencia de productos de tabaco. México: SSA, 2004. [Consultado 2010 octubre]. Disponible en: http:// www.salud.gob.mx/unidades/cdi/ressisi/000 I 200020306_055.pdf

9. Dworak F. Cabildeo, Cuadernos de trabajo. México: Instituto Belisario Domínguez del Senado de la República, 20II.

10. Gaceta Parlamentaria de la Cámara de Diputados, Relación de Cabilderos. México: Cámara de Diputados, 201 I. [Consultado 201 I mayo]. Disponible en: http://gaceta.diputados.gob.mx/Gaceta/6I/20I I/ abr/20I I040I-II.pdf

II. Waters H, Sáenz de Miera B, Ross H, Reynales LM. La economía al tabaco y los impuestos al tabaco en México. París: Unión Internacional contra la Tuberculosis y Enfermedades Respiratorias, 2010:31.
12. Cámara de Diputados. Boletín de Información. México. Cámara de Diputados 2009. [Consultado 2010 marzo]. Disponible en: http://www3. diputados.gob.mx/camara/005_comunicacion/a_boletines/2009_2009/010_ octubre/3I_3I/036I_rechazan_diputados_modificacion_del_senado_ sobre_impuesto_especial_al_tabaco.

13. British American Tobacco México (sitio de internet). México. [Consultado 2010 ene I5]. BATM; (aprox. 6 pantallas) Disponible en: http://www.batmexico.com.mx/OneWeb/sites/BAT_5NNARK.nsf/ vwPagesWebLive/DO7CMNS4? opendocument\&SID $=\& D T C=\& T M P=1$ 14. Maksymiv N. Tabacaleras amenazan con frenar inversiones. CNN Expansión 2010 octubre 20; sección negocios.

15. Organización Mundial de la Salud. Convenio Marco para el Control del Tabaco. Artículo 5.3. Ginebra; OMS, 2003

16. Galarza R. El Negociador, CNN Expansión 2009 noviembre 22; sección negocios. 